\title{
DISCRETE SERIES OF FUSION ALGEBRAS
}

\author{
R. SRINIVASAN, V. S. SUNDER and N. J. WILDBERGER
}

(Received 16 May 2000; revised 24 April 2001)

Communicated by A. H. Dooley

\begin{abstract}
We show that the left regular representation of a countably infinite (discrete) group admits no finitedimensional invariant subspaces. We also discuss a consequence of this fact, and the reason for our interest in this statement.

We then formally state, as a 'conjecture', a possible generalisation of the above statement to the context of fusion algebras. We prove the validity of this conjecture in the case of the fusion algebra arising from the dual of a compact lie group.

We finally show, by example, that our conjecture is false as stated, and raise the question of whether there is a 'good' class of fusion algebras, which contains (a) the two 'good classes' discussed above, namely, discrete groups and compact group duals, and (b) only contains fusion algebras for which the conjecture is valid.
\end{abstract}

2000 Mathematics subject classification: primary 46L37; secondary 43A65.

\section{Introduction}

The considerations of this paper were motivated by a question raised by Popa [6] regarding the spectra of the adjacency matrices of the so-called 'principal graph associated to a subfactor'. Specifically, in case this graph turns out to be infinite, he asked whether the corresponding self-adjoint operator had 'only essential spectrum'.

Just about the only handle one has on the graph-in fact, even the manner in which it is defined, or the boundedness of this operator is proved-is via the fusion rules obeyed गy the various kinds of bimodules that are associated with the subfactor. This fusion llgebra structure was used to show [4] that the spectral measures of the self-adjoint sperators so associated to a subfactor and its 'dual subfactor' are mutually absolutely

c) 2002 Australian Mathematical Society $1446-7887 / 2000 \$ A 2.00+0.00$ 
continuous 'away from 0 '; and that proof depended only on the 'regular representation' of the underlying fusion algebra (albeit, with appropriate ' $M_{2}$-grading'). Motivated by that success, we hoped to deduce the truth of Popa's conjecture from another conjecture about the regular representation of an infinite discrete fusion algebra.

We should mention that there is substantial literature (see [1], for instance) on 'hypergroups', which (at least the discrete ones) are very near relatives of what we call fusion algebras. The simplest examples of these come from discrete groups and the representation theory of compact Lie groups. The 'obvious version' of the desired conjecture turns out to be true in these two special cases, as we show in Proposition 2.1 and Proposition 2.6. (Both these examples also correspond to certain subfactors, so Popa's conjecture is valid for those subfactors.) It turns out however, as demonstrated in Example 2.7, that our 'obvious version' of the conjecture can be false as stated. We still hope/believe that the conjecture would be valid for fusion algebras that satisfy some additional hypotheses which are always met in the case of fusion algebras coming from subfactors.

\section{Main results}

PROPOSITION 2.1. Let $\lambda: G \rightarrow \mathscr{L}\left(\ell^{2}(G)\right)$ denote the left-regular representation of a countably infinite (discrete) group. Then, $\lambda$ does not admit any non-zero finitedimensional sub-representations.

PROOF. Since sub-representations of $\lambda$-the so-called discrete series representations of $G$-are characterised (see [2], for instance) by the property that the associated matrix coefficients yield square-summable functions on $G$, we see that it suffices to prove the following:

Assertion (*) If $\pi: G \rightarrow \mathscr{L}(V)$ is a finite-dimensional unitary representation of $G$, and if $0 \neq \xi \in V$, then we have: $\sum_{t \in G}|\langle\pi(t) \xi, \xi)|^{2}=+\infty$.

Indeed, if $\pi$ is as above, then $C=\overline{\pi(G)}$ is a compact group (by the assumed finitedimensionality of $\pi$ ). Consider the function $f: C \rightarrow \mathbb{C}$ defined by $f(x)=\langle x(\xi), \xi\rangle$. Clearly the function $f$ is continuous and non-zero (as $f(1)=\|\xi\|^{2} \neq 0$ ). Hence if $\epsilon>0$ is sufficiently small, then the set defined by $U=\{x \in C:|f(x)|>\epsilon\}$ is a non-empty open subset of $C$.

We assert that $\pi^{-1}(U)$ is an infinite set in $G$. (This will show that the function $t \mapsto|\langle\pi(t) \xi, \xi\rangle|$ is bounded below by $\epsilon$ on an infinite set, and establish Assertion (*) and complete the proof of the proposition.)

For this, we consider two cases. Firstly, if $\pi(G) \cap U$ is an infinite set, the assertion is obvious. Secondly, suppose $\pi(G) \cap U$ is a finite set. Since $U$ is open and nonempty, and since $\pi(G)$ is dense in $C$, it must be the case that $U$ itself is a finite set; 
since $U$ is a non-empty open set in $C$, and since the compact group $C$ can be covered by finitely many translates of $U$, this implies that $C$ must itself be finite. Since $G$ is infinite, we see that $K=\operatorname{ker} \pi$ must be an infinite subgroup of $G$; finally, pick $\pi\left(t_{0}\right) \in \pi(G) \cap U$-such a $t_{0}$ must exist by density-and note that $t_{0} K \subset \pi^{-1}(U)$; the proof is complete.

COROLlary 2.2. Let $G$ be as in Proposition 2.1, and suppose $T$ is a normal operator in the von Neumann algebra $L G=\lambda(G)^{\prime \prime}$ generated by $\lambda(G)$. (For instance, we might have $T=\lambda(t)$ or $T=\lambda(t)+\lambda\left(t^{-1}\right)$, for any $t \in G$.) Then, $\operatorname{sp} T=\operatorname{sp}_{e} T$, where we write $\mathrm{sp}$ and $\mathrm{sp}_{e}$ to denote the spectrum and the essential spectrum, respectively, of an operator.

ProOF. To start with, it must be noted that the conclusion of Proposition 2.1 is valid, with the left-regular representation $\lambda$ replaced by the right-regular representation $\rho$. (Simply replace $G$ by its 'opposite group' and appeal to the established result.)

To prove the corollary, we need to show that if $\zeta \in \operatorname{sp} T$, then $\zeta \in \operatorname{sp}_{e} T$. So fix such a $\zeta$, and a typical open neighbourhood $U$ of $\zeta$; we need to show then that the associated spectral projection $P=1_{U}(T)$ has infinite rank. Note that $T \in L G$ implies $P \in L G=\rho(G)^{\prime}$, and consequently the range of $P$ yields a sub-representation of $\rho$. Conclude from the first paragraph of this proof that ran $P$ cannot be finite-dimensional, as desired; the proof is complete.

The reason for our interest in the preceding results (apart, of course, from their intrinsic appeal) is that we seek to establish an analogue of the preceding corollary, with 'countably infinite discrete group' replaced by 'fusion algebra with countably infinite basis, which admits a dimension function'. In order to state the 'desired analogue' mentioned above, we need a definition.

DEFINITION 2.3. By a fusion algebra (with countable basis)-which we shall denote by $\mathbb{C} \mathscr{G}$ - we shall mean an associative, unital $*$-algebra over $\mathbb{C}$, equipped with a distinguished (countable) Hamel basis $\mathscr{G}$, whose so-called structure constants $\left\{N_{X Y}^{Z}\right.$ : $X, Y, Z \in \mathscr{G}$, defined by the equations $X Y=\sum_{Z \in \mathscr{G}} N_{X Y}^{Z} Z$ for arbitrary $X, Y \in \mathscr{G}$, are required to satisfy the following conditions, for all $X, Y, Z \in \mathscr{G}$ :

(i) $N_{X Y}^{Z}$ is a non-negative integer; (the Hamel-basis requirement implies that for fixed $X, Y$, we can have $N_{X Y}^{Z}>0$ for at most finitely many $Z$ );

(ii) $\mathscr{G}$ contains the (multiplicative) identity of $\mathbb{C} \mathscr{G}$; and

(iii) there exists an involution $\mathscr{G} \ni X \mapsto \bar{X} \in \mathscr{G}$ such that:

(a) $\bar{X}=X^{*}$; and

(b) $N_{X Y}^{Z}=N_{\bar{X} Z}^{Y}$.

The fusion algebra $\mathbb{C} \mathscr{G}$ is said to admit a dimension function if there exists a function $\mathscr{G} \ni X \mapsto d_{X} \in(0, \infty)$ such that $d_{X} d_{Y}=\sum_{Z \in \mathcal{G}} N_{X Y}^{Z} d_{Z}$. 
Some familiar 'fusion algebras with countable basis, which admit a dimension function' are listed in the following example.

EXAMPLE 2.4. (a) Let $\mathscr{G}=G$ denote a countable group, and let the structure constants, involution, and dimension function be given by:

$$
N_{g h}^{k}=\delta_{g h}^{k}, \quad \bar{g}=g^{-1}, \quad d_{g}=1 \quad \forall g \in G .
$$

(b) For a compact second-countable group $K$, let $\mathscr{G}=\hat{K}$ denote the collection of isomorphism classes of irreducible unitary representations of $K$; define the structure constants, involution, and dimension function by:

$$
\begin{aligned}
N_{\pi \mu}^{\sigma} & =\langle\pi \otimes \mu, \sigma\rangle=\text { multiplicity of } \sigma \text { in } \pi \otimes \mu ; \\
\bar{\pi} & =\text { contragradient representation, } \\
d_{\pi} & =\text { degree of the representation } \pi .
\end{aligned}
$$

(c) If $N \subset M$ is a 'finite-depth subfactor' and if $\mathscr{G}(M, N)$ denotes the set of irreducible $N-N$ bimodules which 'occur' in the tower of Jones' basic construction-see [3], for instance-then $\mathbb{C} \mathscr{G}(M, N)$ is a fusion algebra with countable basis, which admits the dimension function defined by $d_{X}=\operatorname{dim}_{N_{-}}(X)$, the so-called ' $N$-dimension' of the left $N$-module $X$. (The structure constants and the adjoint are defined analogously to (b) above, except that 'tensor-products' and 'contragradients' are to be interpreted appropriately.)

It is not very hard to show-see [4], for instance-that if $\mathbb{C} \mathscr{G}$ is such a 'fusion algebra with countable basis, which admits a dimension function', and if $\ell^{2}(\mathscr{G})$ denotes a (necessarily separable) Hilbert space with orthonormal basis $\left\{\xi_{X}: X \in \mathscr{G}\right\}$ indexed by $\mathscr{G}$, then there exists a unique 'left-regular representation' $\lambda: \mathbb{C} \mathscr{G} \rightarrow \mathscr{L}\left(\ell^{2}(\mathscr{G})\right)$, which is a homomorphism of unital $*$-algebras, and satisfies:

$$
\lambda_{X} \xi_{Y}=\sum_{Z \in \mathscr{G}} N_{X Y}^{Z} \xi_{Z} \quad \forall X, Y \in \mathscr{G} .
$$

This entire discussion was motivated by our desire to affirmatively settle the following conjecture for the case of the examples arising from a subfactor, as in Example 2.4 (c). (Strictly speaking, we want to prove the analogue of Corollary 2.2 , with 'group' replaced by 'fusion algebra' arising as in Example 2.4 (c).)

CONJECTURE. Let $\lambda: \mathbb{C} \mathscr{G} \rightarrow \mathscr{L}\left(\ell^{2}(\mathscr{G})\right)$ denote the left-regular representation of an infinite-dimensional fusion algebra with countable basis, which admits a dimension function. Then, $\lambda$ does not admit any non-zero finite-dimensional sub-representations. 
Actually, we would be happy to settle this conjecture in the case when the fusion algebra satisfies condition (i) in the following lemma, since this condition is always met in our motivating examples (as in Example 2.4 (c)).

LEMMA 2.5. Let $\mathscr{G}=\hat{K}$ be as in Example 2.4 (b). Then the following conditions are equivalent:

(i) $\mathscr{G}$ is 'finitely generated' in the sense that there exists a finite set $S \subset \mathscr{G}$ such that if $\pi \in \mathscr{G}$, then there exist $\rho_{1}, \ldots, \rho_{n}$ in $S$ such that $\left\langle\rho_{1} \otimes \cdots \otimes \rho_{n}, \pi\right\rangle \neq 0$.

(ii) $K$ is isomorphic to a closed subgroup of $U(N)$ for some $N$ (and is, in particular, a Lie group).

Proof. (ii) implies (i). If $\rho: K \rightarrow U(N)$ is a faithful (continuous unitary) representation of $G$, let $\rho_{1}, \ldots, \rho_{n}$ denote the distinct irreducible subrepresentations of $\rho$. Since every irreducible representation of $U(N)$ is contained in some power of the direct sum of the identity representation and its conjugate, the validity of (i) - with $S=\left\{\rho_{1}, \bar{\rho}_{1}, \ldots, \rho_{n}, \bar{\rho}_{n}\right\}$-is seen to follow.

(i) implies (ii). To start with, we assume, as we clearly may, that $S$ is closed under the formation of contragradients. Then, consider the subalgebra of $C(K)$, generated by the matrix entries associated with the representations in $S$. The assumptions show that this is a finitely generated self-adjoint subalgebra which contains the matrix entries of all the irreducible representations of $K$. It follows, then, from the Stone-Weierstrass and Peter-Weyl theorems that the direct sum of the representations in $S$ must be a faithful representation which is finite-dimensional since $S$ is finite.

PROPOSITION 2.6. If $\mathscr{G}=\hat{K}$ is as in the above lemma, and if $K$ is a compact Lie group, then the conjecture is valid for $\mathscr{G}$.

Proof. Suppose $K$ is a compact Lie group. Then $\left\{\chi_{\pi}: \pi \in \hat{K}\right\}$ is an orthonormal set in $L^{2}(K, d g)$; furthermore, the Weyl integration formula shows that there exists a measure-call it $\nu-$ on the maximal torus $\mathbb{T}$ of $K$ such that $\left\{\left.\chi_{\pi}\right|_{T}: \pi \in \hat{K}\right\}$ is an orthonormal set in $L^{2}(\mathbb{T}, v)$. In fact, the linear span $\mathscr{A}_{0}$ of $\left\{\chi_{\pi}: \pi \in \hat{K}\right\}$, is dense, in the uniform norm, in the space of those continuous functions on $\mathbb{T}$ which are invariant under the action of the Weyl group $W$; the latter space can be identified with $C(X)$, where $X$ is any suitable 'fundamental domain' in $\mathbb{T}$ for the $W$-action, which can be chosen to be a 'compact polytope'.

It is thus seen-see any standard text on representation theory, such as [7], for instance-that there exists a compact polytope $X$ (in a sufficiently large dimensional Euclidean space) and a dense unital ${ }^{*}$-subalgebra $\mathscr{A}_{0} \subset C(X)$ such that $\mathbb{C} \mathscr{G} \cong \mathscr{A}_{0}$ (as a *-algebra); and further, the Weyl integration formula shows that there exists a probability measure $\mu$ on $X$ which is mutually absolutely continuous with respect 
to the restriction to $X$ of Lebesgue measure-with a continuous Radon-Nikodym derivative-such that the above isomorphism from $\mathbb{C} \mathscr{G}$ onto $\mathscr{A}_{0}$ extends to a unitary isomorphism of $\ell^{2}(\mathscr{G})$ onto $L^{2}(X, \mu)$. However, all closed subspaces of $L^{2}(X, \mu)$, which are stable under the standard multiplication representation of $C(X)$, are of the form $L^{2}(E, \mu)$ for some subset $E \subset X$ of positive measure. The above description of $\mu$ shows that all such subspaces are infinite-dimensional; and the proof of the proposition is complete.

REMARK. It follows from [5, Theorem 5.1] that if $\mathbb{C} \mathscr{G}$ is a commutative fusion algebra with countable basis and dimension function, then there exists a canonical pair $(X, \mu)$ of a compact Hausdorff space and a probability measure on it, and a *-algebra isomorphism of $\mathbb{C G}$ onto a dense *-subalgebra $\mathscr{A}_{0}$ of $C(X)$ which extends to a unitary isomorphism of $\ell^{2}(\mathscr{G})$ onto $L^{2}(X, \mu)$. It then follows as in the foregoing proof that our conjecture, for this $\mathscr{G}$, is equivalent to the requirement that the measure $\mu$ has no atoms. (This is the most natural route to a proof of Proposition 2.1 in the case of abelian groups.)

We shall now show, by example, that the conjecture, as stated, is false; but we still hope that the conjecture might continue to be valid for all fusion algebras in some restricted class which might contain all the fusion algebras arising from the context of subfactors that initially motivated these considerations.

EXAMPLE 2.7. Let $\mathscr{G}=\left\{X_{n}: n \in \mathbb{Z} \backslash\{0\}\right\} \bigsqcup\left\{h^{k}: 0 \leq k \leq 3\right\}$ with the operations defined as follows:

(a) the set $\left\{h^{j}: 0 \leq j \leq 3\right\}$ is to be thought of as a cyclic group of order 4 ; thus, for example, $\left(h^{j}\right)^{*}=h^{k}$ if $k=-j(\bmod 4)$; and $h^{0}$ is to be the multiplicative identity of the fusion algebra;

(b) we require that $h^{j} \cdot X_{n}=X_{n} \cdot h^{j}=X_{n}$, for every $j$ and $n \neq 0$;

(c) and finally,

$$
X_{n} X_{m}=X_{m} X_{n}= \begin{cases}2 X_{n+m} & \text { if } n \neq-m \\ \sum_{k=0}^{3} h^{j} & \text { if } n=-m,\end{cases}
$$

so that, in particular, we have $X_{n}^{*}=X_{-n}$.

It may be verified that the above definitions equip $\mathbb{C} \mathscr{G}$ with the structure of an infinite-dimensional commutative fusion algebra which has a dimension function satisfying $d_{X_{n}}=2$ and $d_{h^{k}}=1$ for every $n$ and $k$.

Furthermore, the element $\xi=\sum_{k=0}^{3}(-1)^{k} h^{k}$ is seen to define an element of $\ell^{2}(\mathscr{G})$ which spans a 1-dimensional invariant subspace for the left-regular representation of $\mathscr{G}$. In particular, the conjecture is not true for this example. 
QUESTION. Is there a class of fusion algebras, with dimension function, which contains both infinite discrete groups and duals of infinite compact Lie groups, such that the conjecture is valid for all fusion algebras in that class? Specifically, is the conjecture valid for the fusion algebra of ' $N-N$ bimodules which arise in the tower of the basic construction'?

\section{Acknowledgements}

The first author would like to thank Dipendra Prasad for discussions; and the second author would like to thank Kapil Paranjape for his help in arriving at the proof presented here for Proposition 2.1.

\section{References}

[1] W. Bloom and H. Heyer, Harmonic analysis of probability measures on hypergroups (de Gruyter, Berlin, 1995).

[2] J. Dixmier, $C^{*}$-algebras (North-Holland, Amsterdam, 1981).

[3] V. F. R. Jones and V. S. Sunder, Introduction to subfactors (Cambridge University Press, Cambridge, 1997).

[4] V. Kodiyalam and V. S. Sunder, 'Spectra of principal graphs', Internat. J. Math. to appear.

[5] N. Obata and N. J. Wildberger, 'Generalized hypergroups and orthogonal polynomials', Nagoya Math. J. 142 (1996), 67-93.

[6] S. Popa, Private communication.

[7] V. S. Varadarajan, Lie groups, Lie algebras and their representations (Springer, New York, 1984).

Indian Statistical Institute

R.V. College Post

Bangalore 560059

India

e-mail: srinivasan.raman@

labomath.univ-orleans.fr
The Institute of Mathematical Sciences

Taramani

Chennai 600113

India

e-mail: sunder@imsc.ernet.in

University of New South Wales

Sydney NSW 2052

Australia

e-mail: n.wildberger@unsw.edu.au 
